\title{
CARACTERÍSTICAS CLÍNICAS Y SOCIODEMOGRÁFICAS DE PACIENTES CON ANEMIA MEGALOBLÁSTICA HOSPITALES DE SAN JOSÉ E INFANTIL UNIVERSITARIO DE SAN JOSÉ
}

José Ignacio Hernández Cruz MD*, Juan José Diaztagle Fernández MD**, Javier Enrique Bolaño Cantillo MD***, José Fernando Castañeda Fierro MD***, Edna Carolina Araque Parra MD****

\section{Resumen}

La deficiencia de vitamina B 12 es la causa más frecuente de anemia después del origen ferropénico. Objetivo: establecer el perfil sociodemográfico y clínico en anemia megaloblástica. Materiales y métodos: serie de pacientes hospitalizados con anemia megaloblástica entre enero 2010 y diciembre 2011 en los hospitales de San José e Infantil Universitario de San José, Bogotá DC. Resultados: 17 casos, 11 en hombres. Edad promedio 59 años (DE: 15.8 años, rango: $37-82$ ), $88 \%$ casados, estrato dos $76.4 \%$, pensionados $17 \%$, con ingresos de dos salarios mínimos legales vigentes mensuales $94.1 \%$ y conviven con más de dos personas $75.4 \%$. Siete pacientes (41.1\%) con déficit combinado de ácido fólico y vitamina B12, de vitamina B 12 tres (17.6\%) y de ácido fólico dos $(\mathbf{1 4 . 2 \%})$. Las manifestaciones más frecuentes fueron anorexia $(\mathbf{7 0 . 5 \%})$, piel seca $(62.5 \%)$, glositis atrófica $(29,4 \%)$ e ictericia (23.5\%). Se observó asociación con enfermedad autoinmune en 17.6\%, neoplasias de origen no hematológico $11.7 \%$ y consumo crónico de alcohol $35.3 \%$. Conclusión: la mayoría pertenecieron a estrato socioeconómico bajo, con ingresos limitados y familias constituidas por más de dos personas. Las manifestaciones más frecuentes fueron digestivas, dermatológicas y neurológicas. Se encontró déficit combinado de vitamina B12 y ácido fólico, exclusivo de ácido fólico asociado con consumo crónico de alcohol y solo de vitamina B 12. Palabras clave: anemia megaloblástica, metabolismo, vitamina B12, ácido fólico, deficiencia.

Abreviaturas: AM, anemia megaloblástica.

\section{CLINICAL, SOCIAL AND DEMOGRAPHIC CHARACTERISTICS OF PATIENTS WITH MEGALOBLASTIC ANEMIA - SAN JOSÉ AND INFANTIL UNIVERSITARIO DE SAN JOSÉ HOSPITALS}

\section{Abstract}

Vitamin B12 deficiency is the second leading cause of anemia following iron deficiency. Objective: to establish the social and demographic profile of megaloblastic anemia. Materials and Methods: series of inpatients diagnosed with mega-

Fecha recibido: abril I 3 de 2013 - Fecha aceptado: mayo 24 de 2013

* Jefe del Servicio de Medicina Interna, Hospital de San José. Profesor Emérito, Fundación Universitaria de Ciencias de la Salud. Bogotá DC, Colombia.

** Especialista en Medicina Interna, epidemiólogo, magister en fisiología. Instructor Asistente, Fundación Universitaria de Ciencias de la Salud. Bogotá DC, Colombia.
*** Especialista en Medicina Interna, Fundación Universitaria de Ciencias de la Salud. Bogotá DC, Colombia.

***** Residente I de Medicina Interna, Fundación Universitaria de Ciencias de la Salud. Bogotá DC, Colombia. 
loblastic anemia admitted to San José and Infantil Universitario de San José hospitals, Bogotá DC, between January 2010 and December 2011. Results: of the 17 cases included 11 were men; mean age was 59 years (SD: 15.8 years, range: 37-82), $88 \%$ were married, $76.4 \%$ belonged to level two socioeconomic stratum, $17 \%$ were retired, $94.1 \%$ earned two legal minimum monthly salaries and $75.4 \%$ lived with more than two family members. Seven patients $(\mathbf{4 1 . 1 \% )}$ had a combined deficiency of folic acid and vitamin B12, three (17.6\%) only a vitamin B12 deficiency and two (14.2\%) only a folic acid deficiency. The most frequent manifestations were anorexia (70.5\%), dry skin (62.5\%), atrophic glossitis $\mathbf{2 9 . 4 \%}$ ) and jaundice (23.5\%). Association with an autoimmune disease was seen in $\mathbf{1 7 . 6 \%}$, with non-hematologic neoplasms $11.7 \%$ and with chronic alcohol abuse $35.3 \%$. Conclusion: most patients belonged to a low socioeconomic stratum with limited income and families with more than two members. The most frequent manifestations were digestive, dermatologic and neurological disorders. Combined deficiency of vitamin B12 and folic acid, only of folic acid associated with chronic alcohol abuse and only of vitamin B12 was found.

Key words: megaloblastic anemia, metabolism, vitamin B12, folic acid, deficiency.

\section{Introducción}

La deficiencia de vitamina B 12 es la causa más frecuente de anemia en nuestro medio, después del síndrome anémico de origen ferropénico. ${ }^{1}$ A nivel mundial, la prevalencia varía entre 5 y $60 \%$ en adultos mayores. La incidencia de la enfermedad es de 1:10.000 en países industrializados, ocurre en todas las razas, con un pico entre los 60 y 70 años, y relación mujer:hombre de 1.6:1.0.,3

La vitamina B12 (cobalamina) juega un papel importante en la síntesis de DNA y en la maduración celular. El organismo no es capaz de producirla por lo que debe ser aportada en los alimentos (carnes, leche, huevos, pescados). Su absorción ocurre en el íleon distal y luego pasa a la circulación unida a la trascobalamina II, que la transporta al hígado y otras zonas del organismo para ejercer su acción. Los requerimientos mínimos son alrededor de 2 a $3 \mu \mathrm{g}$ diarios y las reservas corporales al igual que la circulación enterohepática ahorran gran parte de la vitamina, suficiente para cubrir los requerimientos luego de un período de tres a cuatro años con déficit en el aporte vitamínico. ${ }^{4-6}$

El ácido fólico por su parte, es una vitamina del complejo B que se sintetiza por las bacterias de la flora intestinal y es proporcionada en pequeñas cantidades por los alimentos (frutas, verduras, lácteos, cereales, entre otros), se absorbe en gran parte en el yeyuno y es convertido en poliglutamatos, lo que permite su permanencia en el interior de las células del organismo. Las necesidades diarias van de 50 a $100 \mu \mathrm{g}$. Su reserva es escasa por lo que su deficiencia se presenta luego de cuatro meses de carencia en el aporte.,.$^{3,8}$

Los cambios morfológicos propios de la AM se presentan en las tres líneas celulares medulares (glóbulos rojos, blancos y plaquetas), como también en otras líneas celulares no hematopoyéticas con elevado recambio celular (piel, mucosas, epitelio gastrointestinal). ${ }^{2}$ Dentro de las causas de AM se encuentran afecciones gástricas, intestinales, insuficiencia pancreática, dieta vegetariana estricta e historia familiar de ésta o enfermedades asociadas.

El envejecimiento y los cambios funcionales propios hacen que los adultos mayores sean más susceptibles a estados de malnutrición y a deficiencias específicas. ${ }^{9}$ Estudios nutricionales realizados en India, Israel, China y Latinoamérica han establecido que entre un 5\% y $15 \%$ de los ancianos tienen deficiencia de vitamina B12. ${ }^{10}$

La presentación clínica de la AM es insidiosa con síntomas inespecíficos como astenia, disminución de la capacidad de concentración y déficit cognitivo, cefalea y síntomas cardiovasculares (palpitaciones y dolor torácico). Menos frecuentes son las manifestaciones neurológicas como parestesias, marcha inestable, espasticidad, neuropatía periférica, degeneración axonal e incluso muerte neuronal, por lo cual es importante 
reconocerlas de manera temprana ya que pueden dejar secuelas permanentes. ${ }^{11}$

En nuestro medio no se conocen con claridad las características clínicas y sociodemográficas de los pacientes con AM lo cual es importante aclarar, dada la relación de la patología con los factores nutricionales. El objetivo de este trabajo es describir el perfil sociodemográfico y clínico que caracterizó a la población de pacientes con AM que acudieron a los hospitales de San José e Infantil Universitario de San José entre enero 2010 y diciembre 2011.

\section{Materiales y métodos}

El tipo de estudio corresponde a una serie de pacientes con diagnóstico de AM definida por los valores de hemoglobina (hombres $\leq 13 \mathrm{~g} \%$ y mujeres $\leq 12 \mathrm{~g} \%$ ), hematocrito ( $\leq 39 \%$ en hombres $\mathrm{y} \leq 36 \%$ en mujeres) y macrocitosis (VCM $>100 \mathrm{pg} / \mathrm{dl}$ ), asociados con déficit de vitamina $\mathrm{B} 12(<250 \mathrm{mcg} / \mathrm{dl})$, ácido fólico ( $<20 \mathrm{mcg} / \mathrm{dl}$ ) o ambos, confirmados mediante método de quimioluminiscencia amplificada (técnica estandarizada) o con alteraciones típicas de esta patología en el extendido de sangre periférica. ${ }^{6,12-14}$ Se incluyeron todos los pacientes mayores de 30 años que se encontraban hospitalizados con este diagnóstico, tanto casos nuevos como en tratamiento entre enero 2010 y diciembre 2011. Las características sociodemográficas, clínicas, paraclínicas, endoscópicas e histopatológicas fueron obtenidas mediante entrevista con el paciente, revisión directa de resultados y de los registros anotados en las historias clínicas. Se excluyeron aquellos que padecían en el momento de su evaluación enfermedades que modificaban su expectativa de vida en los siguientes meses (cáncer terminal, receptores de quimio ó radioterapia) o con diagnóstico de enfermedades mielo y linfoproliferativas. Se indagó sobre ingesta crónica de alcohol, el número de veces por semana y los años de consumo.

Los parámetros hematológicos fueron medidos mediante técnica automatizada en equipo Coulter LH 750 automático, calibrado y certificado por
ICONTEC con las medidas internacionales, para garantizar un margen de error de 0.01 unidades en los resultados.

Todos los datos fueron procesados en Microsoft Excel, la calidad de los mismos fue revisada en su totalidad y el análisis estadístico se hizo en el programa stata10. ${ }^{\circledR}$. El protocolo fue revisado y aprobado por el Comité de Investigaciones y Ética de la Facultad de Medicina de la Fundación Universitaria de Ciencias de la Salud, se consideró que no representaba riesgo para los participantes por lo que no requirió consentimiento informado.

\section{Resultados}

Se describen 17 pacientes con AM, los cuales fueron tratados en el servicio de medicina interna. Se presentaron once hombres y seis mujeres. Cinco ya tenían diagnóstico y venían recibiendo tratamiento. Todos cursaban con macrocitosis aunque solo tres persistían con anemia.

La edad promedio fue 59 años (DE: 15.8 años, rango: 37-82), el 88\% casados, pertenecían al estrato dos el $76.4 \%$, pensionados $17 \%$, habitantes en el área metropolitana de Bogotá DC $88.2 \%$, con ingresos de dos salarios mínimos legales vigentes mensuales $94.1 \%$ y convivían con más de dos personas en su domicilio $75.4 \%$ (Tabla 1).

Las manifestaciones clínicas más frecuentes fueron anorexia $(70.5 \%)$, piel seca $(62.5 \%)$, glositis atrófica $(29,4 \%)$ e ictericia $(23.5 \%)$. Se observó con menos frecuencia queilitis $(11.7 \%)$, parestesias y pérdida de la función muscular (11.7\%). Las principales comorbilidades encontradas fueron hipotiroidismo (29.4\%), enfermedad autoinmune (17.6\%), neoplasias de origen no hematológico $(11.7 \%)$ y diarrea crónica $(5.8 \%)$. Se reportó consumo crónico de alcohol (35.3\%) y dieta vegetariana $(5.8 \%)$ (Tabla 2 ).

Se realizó endoscopia de vías digestivas altas a 14 pacientes, confirmándose gastritis atrófica por biopsia en 10 casos 71.4\%, (reporte final de gastritis atrófica multifocal o crónica atrófica). Se encontró un paciente 


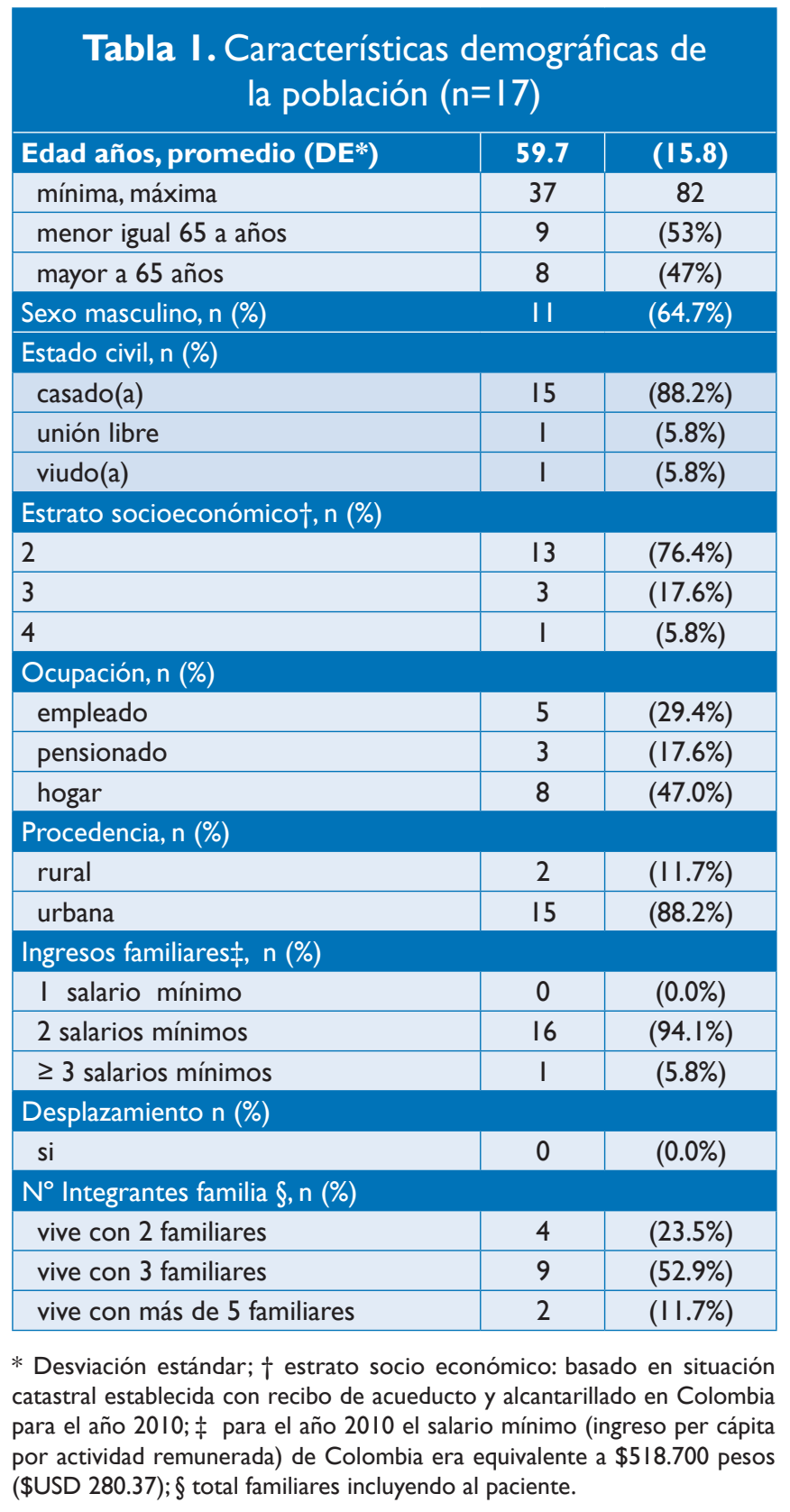

adulto mayor (74 años), con anemia severa (Hb de 3,5 gr, Hcto de 10\%), macrocitosis (VCM de 114), trombocitopénico, con déficit combinado de vitamina B12 y ácido fólico, antecedente de alto consumo de alcohol durante gran parte de su vida y endoscopia normal.

Se encontraron ocho pacientes $(47 \%)$ con déficit combinado de ácido fólico y vitamina B12, de los cuales cinco pacientes mayores de 65 años. El déficit exclusivo de vitamina B 12 se identificó en dos pa-

\begin{tabular}{|l|c|}
\hline \multicolumn{3}{|l|}{ Tabla 2. Características clínicas de la población } \\
$(\mathrm{n}=\mid 7)$
\end{tabular}

* Datos de 14 pacientes. Hallazgos EVDA confirmados por biopsia; $†$ I caso de artritis reumatoide, I de lupus eritematoso y I de tiroiditis.

cientes (11.7\%) y de ácido fólico puro en uno (5.8\%). No hubo información de resultados de ácido fólico en dos casos. Durante el estudio se documentaron cuatro pacientes $(23.5 \%)$ que cumplían criterios de inclusión por anemia y macrocitosis, sin hallar déficit de vitamina B 12 ni de ácido fólico pero con niveles en el límite inferior $(260-280 \mathrm{pg} / \mathrm{dl})$. Entre aquellos con déficit de ácido fólico $\mathrm{n}=9$ (ya sea combinado o exclusivo) cinco tenían antecedentes de consumo crónico de alcohol.

Se hizo una comparación entre los resultados de las variables de hemoglobina, hematocrito, leucocitos, plaquetas, valores de vitamina B12 y ácido fólico entre el grupo en que se halló anemia de novo y el grupo que ya tenía diagnóstico conocido de AM (Tabla 3). 


\begin{tabular}{|c|c|c|c|c|c|c|}
\hline & \multicolumn{3}{|c|}{ Anemia de novo $(n=12)$} & \multicolumn{3}{|c|}{ Anemia ya diagnosticada $(n=5)$} \\
\hline & Mediana & RIQ & Min-max & Mediana & RIQ & Min-max \\
\hline Hemoglobina gr\% & 9.35 & $6.25-11.55$ & $3.5-12.8$ & 12.2 & $8.5-13.6$ & $7.2-15.2$ \\
\hline Hematocrito (\%) & 27.55 & $39.25-65.85$ & $10.2-39.9$ & 38.9 & $24.6-42.0$ & $21.2-42.0$ \\
\hline Leucocitos (leuc/mm3) & 4.505 & $3.925-6.585$ & $1.900-9.800$ & 4.200 & $4.100-5.920$ & $4.100-6.730$ \\
\hline Plaquetas (plaq /mm3) & 209.500 & $\begin{array}{l}42.600- \\
349.500\end{array}$ & $\begin{array}{l}17.000- \\
580.000\end{array}$ & 255.000 & $\begin{array}{l}102.000- \\
267.000\end{array}$ & $50.000-429.700$ \\
\hline Niveles de vitamina BI2 (pg/dl) & 174.3 & $65.9-279$ & $2.82-1001$ & 78 & $52.3-157$ & $42.2-260$ \\
\hline Acido fólico* (pg/dl) & 15.2 & $10.4-25$ & $5.8-25$ & 19.75 & $14.25-20$ & $5.8-25$ \\
\hline
\end{tabular}

* Datos disponibles en nueve pacientes con diagnóstico de novo y cuatro con anemia conocida.

En el extendido de sangre periférica de los pacientes con AM de novo, los hallazgos más significativos fueron macrocitosis $72.7 \%$, anisocitosis $75.0 \%$, policromatofilia 50\%, hipercromía 40.\%, esferocitosis $33.3 \%$ y esquistocitosis $33.3 \%$. En aquellos con diagnóstico conocido no se realizó frotis de sangre periférica. Cuatro pacientes con anemia de novo $(43.8 \%)$ requirieron soporte transfusional con glóbulos rojos empaquetados y uno solo de glóbulos rojos y plaquetas. En ninguno de los casos se realizó la medición de niveles de anticuerpos antifactor intrínseco.

\section{Discusión}

Nuestro estudio permitió observar el perfil sociodemográfico predominante en los hospitales de San José e Infantil Universitario de San José, el cual fue el de paciente masculino que vivía en estrato social dos, pensionado y con ingresos que no superaban los dos salarios mínimos legales vigentes, con un núcleo familiar constituido por más de dos personas. Se logró establecer una edad mediana de 59 años que se correlacionó con las modificaciones en los hábitos nutricionales en este grupo etáreo, con las características socioeconómicas mencionadas y con las alteraciones anatomopatologicas encontradas.

Cuatro de los pacientes que ingresaron al estudio cumpliendo con criterios de inclusión dados por anemia y macrocitosis no tenían déficit de ácido fólico ni de vitamina B 12. Existen reportes en la literatura que indican que la medición de niveles de vitamina B12 puede no ser fiable para detectar la deficiencia y la medida de homocisteina y/o ácido metilmalónico debe usarse para confirmarla en pacientes asintomáticos de alto riesgo con valores normales de vitamina B12. ${ }^{12}$ Esta medición no la realizamos.

En el presente estudio encontramos que como lo menciona la literatura, la deficiencia de cobalamina (vitamina B12) causó alteraciones hematológicas y neurológicas. Entre las primeras fueron anemia, macrocitosis y aparición de esquistocitos, eliptocitos y neutrófilos hipersegmentados en el frotis de sangre periférica. Las manifestaciones neurológicas incluyeron parestesias y disminución o pérdida de la fuerza muscular, lo cual también concuerda con las descripciones publicadas.

Fue menos frecuente la AM por déficit de ácido fólico puro, pero en los casos en los que se evidenció, su comportamiento fue más severo respecto a las manifestaciones neurológicas.

Otras investigaciones también han evaluado las causas, mecanismos, características clínicas y métodos diagnósticos utilizados en AM. Una serie de 52 casos en China mostró resultados similares a los nuestros, aunque la muestra fue más grande y se estudiaron los niveles de anticuerpos contra factor intrínseco y células parietales gástricas. ${ }^{15}$

Los hallazgos de este estudio demostraron que la medición sérica de niveles de vitamina B12 y ácido 
fólico estandarizadas como único método de detección de déficit en nuestro país, permite establecer niveles bajos de manera temprana antes de que aparezcan las alteraciones en el volumen corpuscular medio y que desciendan los valores de hemoglobina y/o hematocrito, e incluso antes de la aparición de la sintomatología propia de anemia. Sin embargo, como ya se mencionó, si quisiéramos ser un poco más precisos y anticiparnos a los síntomas, se podrían realizar mediciones de homocisteina y ácido metilmalónico, los cuales cambian incluso antes que los niveles de cobalamina. ${ }^{12,16}$

En el estudio no fue posible en todos los casos realizar un nuevo interrogatorio a los pacientes que presentaban datos parciales en los registros de la historia clínica y no hubo cobertura en la medición de niveles de anticuerpos anti-factor intrínseco por parte de las aseguradoras de los pacientes, por lo que no se pudo contar con este dato.

\section{Conclusión}

En esta serie de casos, la mayor parte de los pacientes pertenecieron a estrato socioeconómico bajo, con ingresos limitados y familias constituidas por más de dos personas. Las manifestaciones más frecuentes fueron digestivas, dermatológicas y neurológicas. Se encontró déficit combinado de vitamina B12 y ácido fólico, déficit exclusivo de ácido fólico asociado con consumo crónico de alcohol y déficit de vitamina B 12.

\section{Referencias}

1 De Paz R, Hernandez-Navarro F. Manejo, prevención y control de la anemia megaloblástica secundaria a déficit de ácido fólico. Nutr. Hosp. 2006 Ene-Feb. 21(1): 113-9.

2 Andres E, Loukili NH, Noel E, Kaltenbach G, Abdelgheni MB, Perrin AE, et al. Vitamin B12 (cobalamin) deficiency in elderly patients. CMAJ 2004;171(3):251-9.

3 Babior B. Folate, cobalamin, and megaloblastic anemias. In: Lichtman MA, Kipps TJ, Seligsohn U, Kaushansky K, Prchal JT, editors. Williams Hematology. 7th ed. New York, McGraw-Hill; 2006. p. 477-509.

4 Chaney SG. Macronutrients: metabolic effects and health Implications. In: Devlin TM. Text of Biochemistry with clinical correlation. 5th ed. New York: Wiley; 2004. p. 1154-7.

5 Binder RA. Nutrient digestion and absorption. In: Boron WF, Boulpaep EL, editors. Medical Physiology: a cellular and molecular approach. 2nd ed. Philadelphia : Saunders : Elsevier;2009. p. 968-76.

6 Babior B, Bunn H. Megaloblastic anemias. In: Kasper DL, et al, editors. Harrison's principles of internal medicine. 16th ed. New York: McGraw-Hill; 2005. pp. 601-07.

7 Anemia megaloblástica y otras causas de macrocitosis. En: Sans-Sabrafen J. Hematologia clínica. 5a ed. Madrid, España: Elsevier;2006. p. 163-86.

8 Dali-Youcef N, Andrès E. An update on cobalamin deficiency in adults. QJM. 2009; 102(1):17-28.

9 Lahner E, Annibale B. Pernicious anemia: new insights from a gastroenterological point of view. World J Gastroenterol. 2009; 15(41):5121-8.

10 Kaferle J, Strzoda CE. Evaluation of macrocytosis. Am Fam Physician. 2009; 79(3):203-8

11 Shah A. Megaloblastic anemia--Part II. Indian J Med Sci. 2004 Jul;58(7):309-11.

12 Antony A. Megaloblastic anemias. In: Hoffman R, et al. edit. Hoffman: hematology: basic principles and practice. 6th ed. Philadelphia, Saunders: Elsevier; 2012.

13 Kaferle J. Evaluation of macrocitosis. Am Fam Phys. 2009; 79(3): 203-8.

14 Cattan D. Pernicious anemia: what are the actual diagnosis criteria? World J Gastroenterol. 2011; 17(4):543-4

15 Chan JC, Liu HS, Kho BC, Chu RW, Ma ES, Ma KM, et al. Megaloblastic anaemia in Chinese patients: a review of 52 cases. Hong Kong Med J. 1998 Sep; 4(3):269-74

16 Langan RC, Zawistoski KJ. Update on Vitamin B12 Deficiency. Am Fam Physician. 2011 Jun 15; 83(12):1425-30. 